\title{
Clinical and radiological evaluation of allograft material in GBR procedures.
}

IMPLANT THERAPY OUTCOMES, SURGICAL ASPECTS
Alexey Ryabov¹, Nikolay Makarov¹, Mikhail Lekishvili²

1Private practice, Moscow Russia

${ }^{2}$ Central Institute of Traumatology and Orthopaedics, Moscow Russia

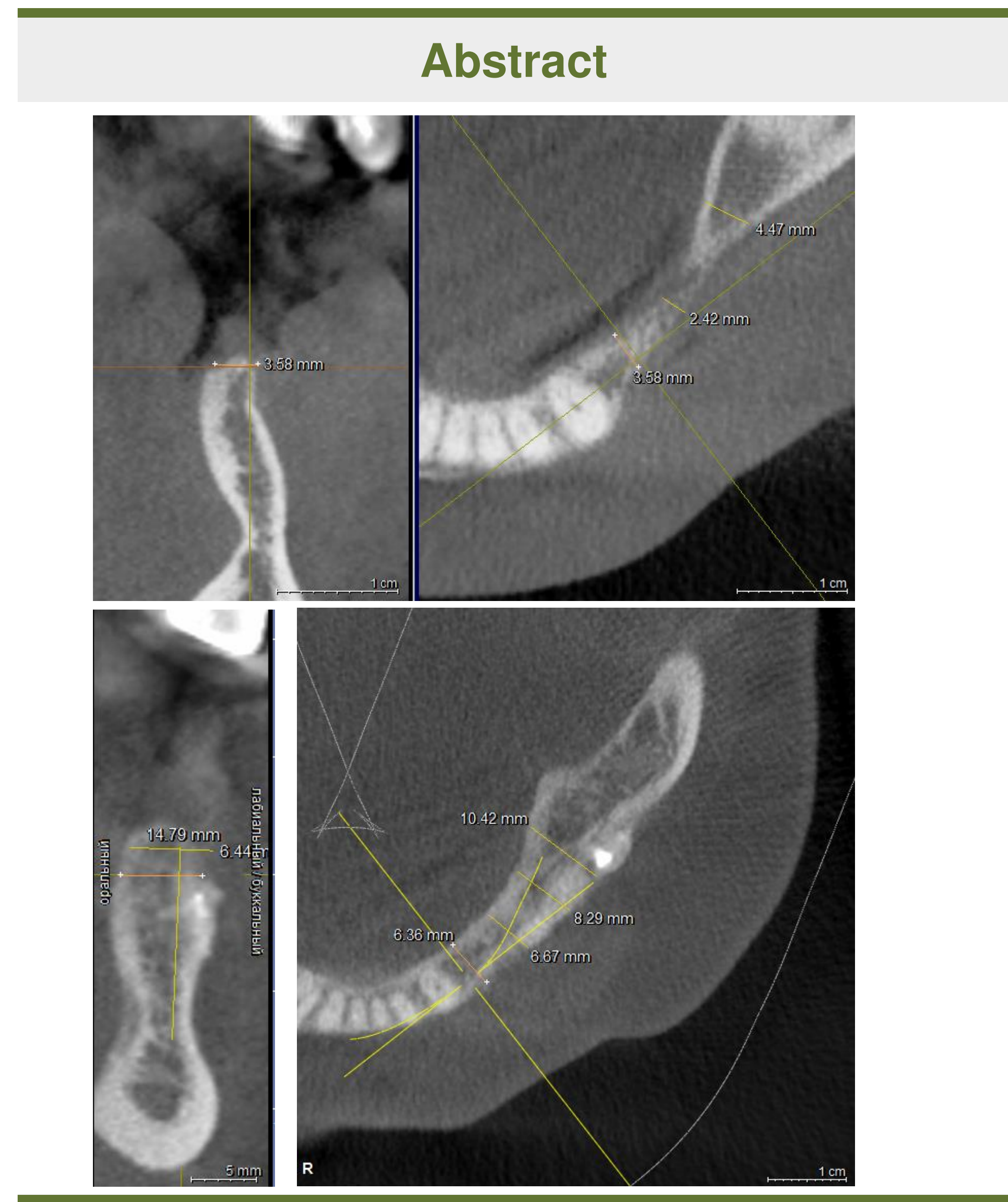

Background and Aim

The cases with horizontal bone atrophy can be treated with different surgical techniques and materials to create adequate bone volume for eventual implant placement. The main differences of these techniques are in the results achieved: bone quality and graft preservation. Allograft materials can format a completely vital bone and preserve the achieved volume from baseline of implant placement to long term period.

The aim of the study was to evaluate the regenerated bone volume and its stability in 5 years.

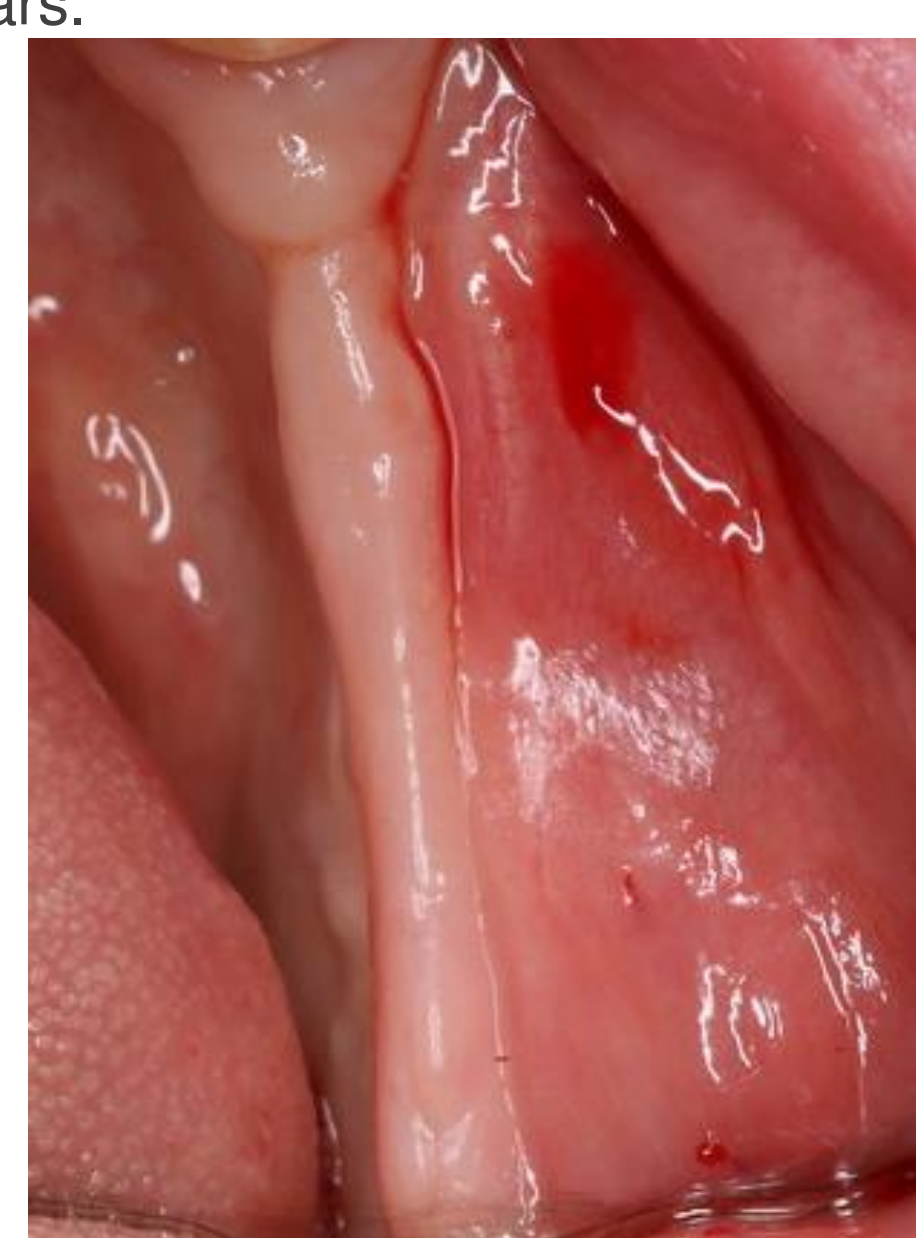

\section{Methods and Materials}

Forty patients with horizontal bone defects with the length of up to three missing teeth were included. Patients were divided in two groups. In the first group patients with atrophy in the maxilla were divided in two subgroups: with anterior and posterior defects. In the second group patients with atrophy in the mandible were divided in two subgroups: with anterior and posterior defects. All patients undergone two-stage procedures. First they received horizontal GBR procedure with "Perfoost" Demineralized Bone Allograft material. Clinical and radiological (CBCT) evaluation were performed before GBR and at implant placement, radiological (CBCT) evaluation after 5 years.

\section{Results}

In total 72 Straumann TL implants were placed. In 1st group 1st subgroup regenerated bone volume resulted in $4,6+/-0,7 \mathrm{~mm}$, 2nd subgroup in 4,8+/-0,9 mm. In 2 nd group 1 st subgroup regenerated bone volume resulted in 4,4+/-0,4 $\mathrm{mm}$, 2nd subgroup in 4,3+/-0,6 $\mathrm{mm}$. The data was not statistically significant. The study was initiated in 2010. After 5 years at radiological (CBCT) evaluation promising results were obtained, and are now being processed.

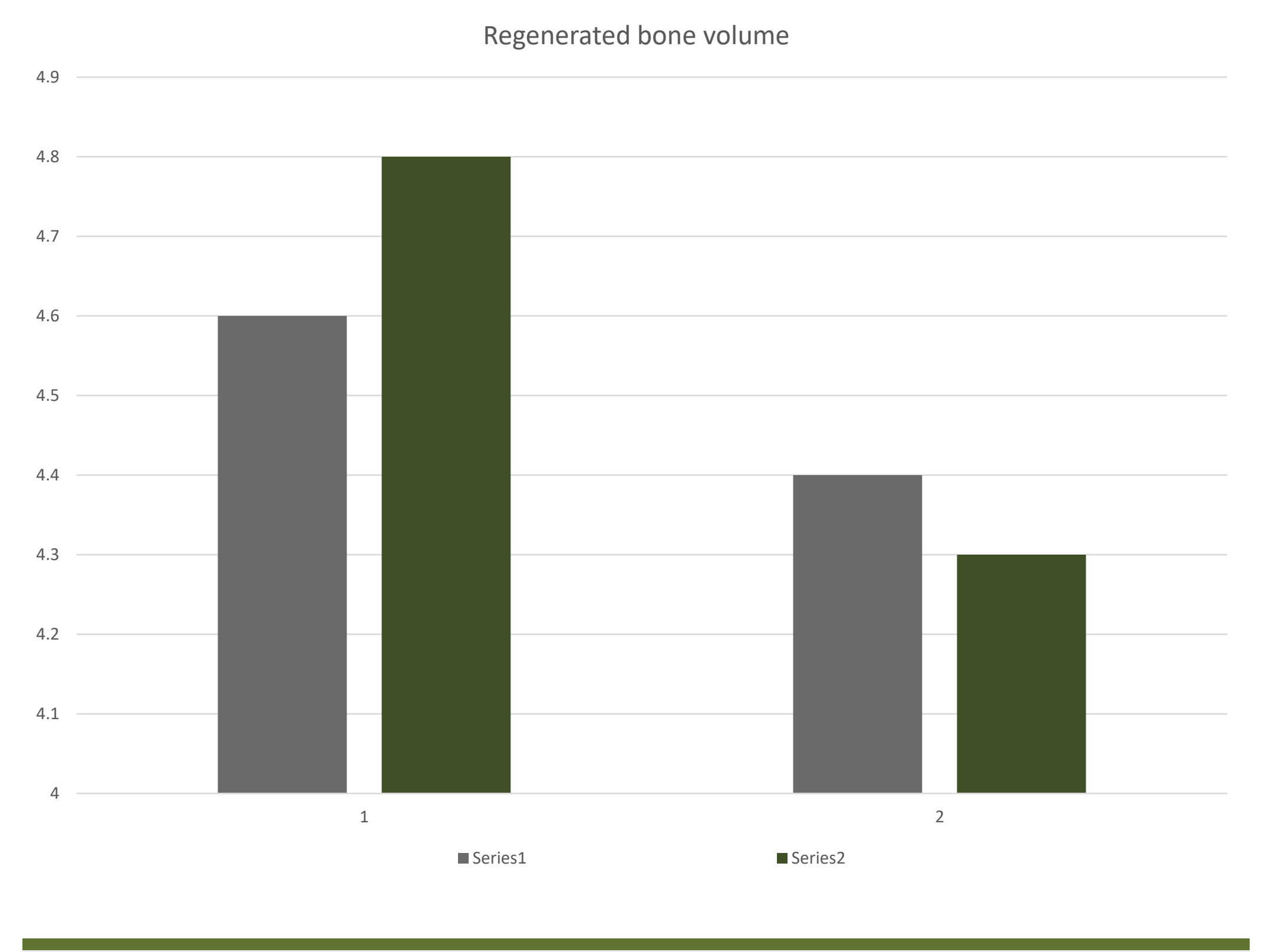

\section{Conclusions}

Results of the study demonstrate high effectiveness of allograft materia in obtaining vital bone regenerate and its high volume stability in long term. Thus we can presume that allograft materials can be an alternative to autologous bone in GBR procedures. Further studies comparing these two techniques are needed.
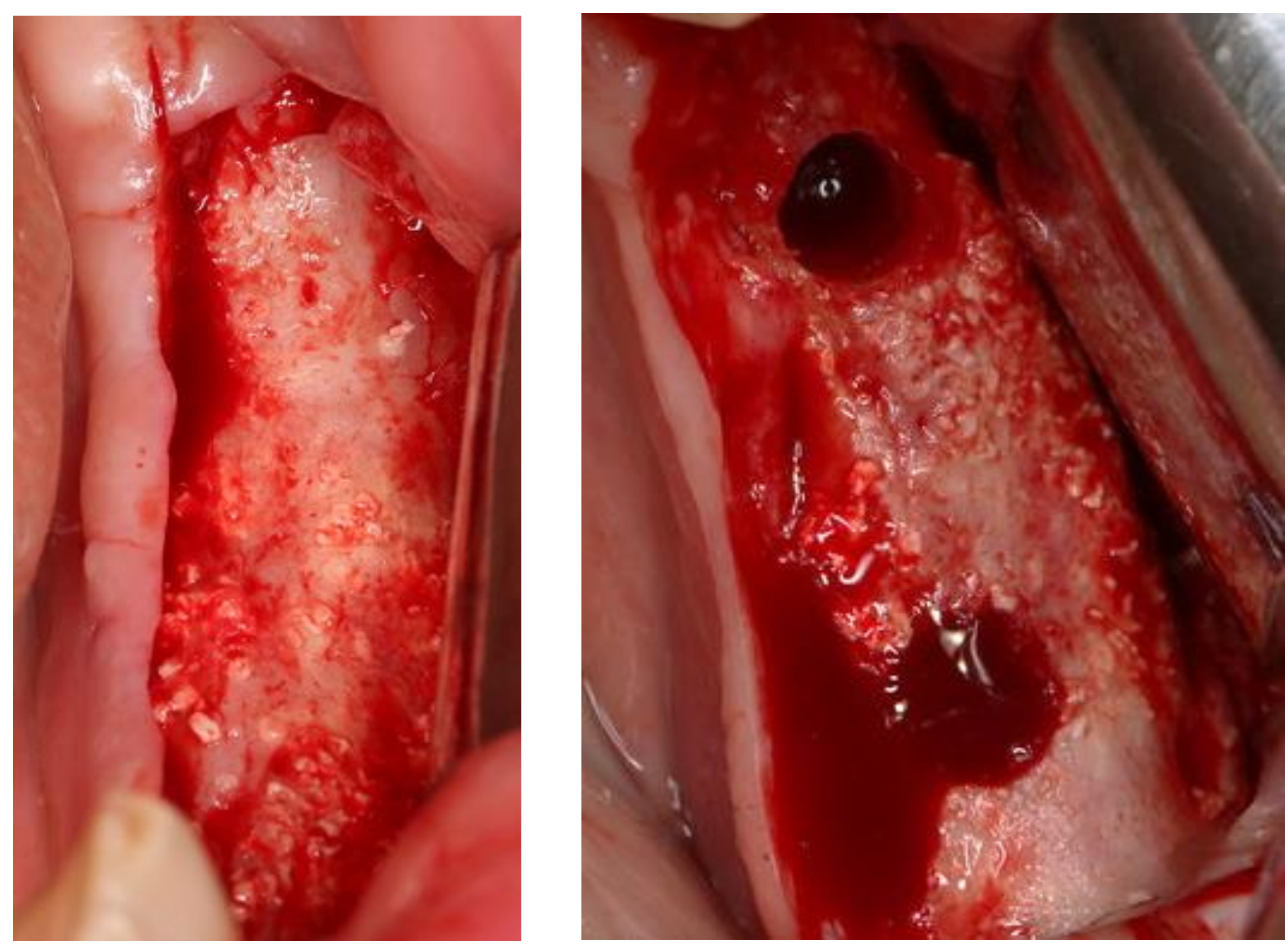

\section{References}

1. Gomes KU, Carlini JL, Biron C, Rapoport A, Dedivitis RA. Use of allogeneic bone graft in maxillary reconstruction for installation of dental implants. J Oral Maxillofac Surg. 2008 Nov;66(11):2335-8.

2. Deluiz D, Oliveira L, Fletcher P, Pires FR, Nunes MA, Tinoco EM. Fresh-Frozen Bone Allografts in Maxillary Alveolar Augmentation:

Analysis of Complications, Adverse Outcomes, and Implant Survival. J Periodontol. 2016 Nov;87(11):1261-1267. Epub 2016 Jul 1.

3. Urist MR. Bone: Formation by autoinduction. Science 150:893, 1965 